\title{
Techniques et culture : les bases d'un programme de travail
}

Techniques and culture: bases for workprogram

\section{Robert Cresswell}

\section{(2) OpenEdition \\ 1 Journals}

Édition électronique

URL : https://journals.openedition.org/tc/4979

DOI : $10.4000 /$ tc. 4979

ISSN : 1952-420X

Éditeur

Éditions de l'EHESS

\section{Édition imprimée}

Date de publication : 30 juin 2010

Pagination : 20-45

ISSN : 0248-6016

\section{Référence électronique}

Robert Cresswell, «Techniques et culture : les bases d'un programme de travail », Techniques \& Culture [En ligne], 54-55 | 2010, mis en ligne le 30 juin 2013, consulté le 29 septembre 2022. URL : http:// journals.openedition.org/tc/4979; DOI : https://doi.org/10.4000/tc.4979 
Techniques E Culture 54-55 volume 1, 2010 : 23-45

\section{TECHNIQUES ET CULTURE}

\section{Les bases d'un programme de travail}

À l'ouverture de cette suite de publications il semble opportun d'expliciter les prémisses de nos recherches. Ce qui suit est donc un résumé des concepts, des hypothèses de travail, des thèmes d'étude qui ont provoqué la constitution de notre groupe de travail, et un aperçu sur l'état des connaissances actuelles dans ce domaine.

\section{Conceptualisation}

\section{Thématique}

Le postulat de base, celui qui ne sera jamais mis en doute, est qu'il existe des rapports entre phénomènes techno-économiques et manifestations socio-culturelles. Le maniement gestuel d'un outil, l'agencement d'une chaîne opératoire, l'intégration de différentes chaînes opératoires au sein d'un groupe humain, le rendement d'une technique de transformation, pour ne parler que de certains aspects ne sont pas des phénomènes à finalité limitée, interne, mais des phénomènes qui influencent de façon directe ou indirecte, ponctuelle ou diffuse, les comportements sociaux et individuels des membres d'un groupe. Ils agissent en tant que supports des rapports sociaux d'un processus économique de production, ou encore en tant que créateurs 

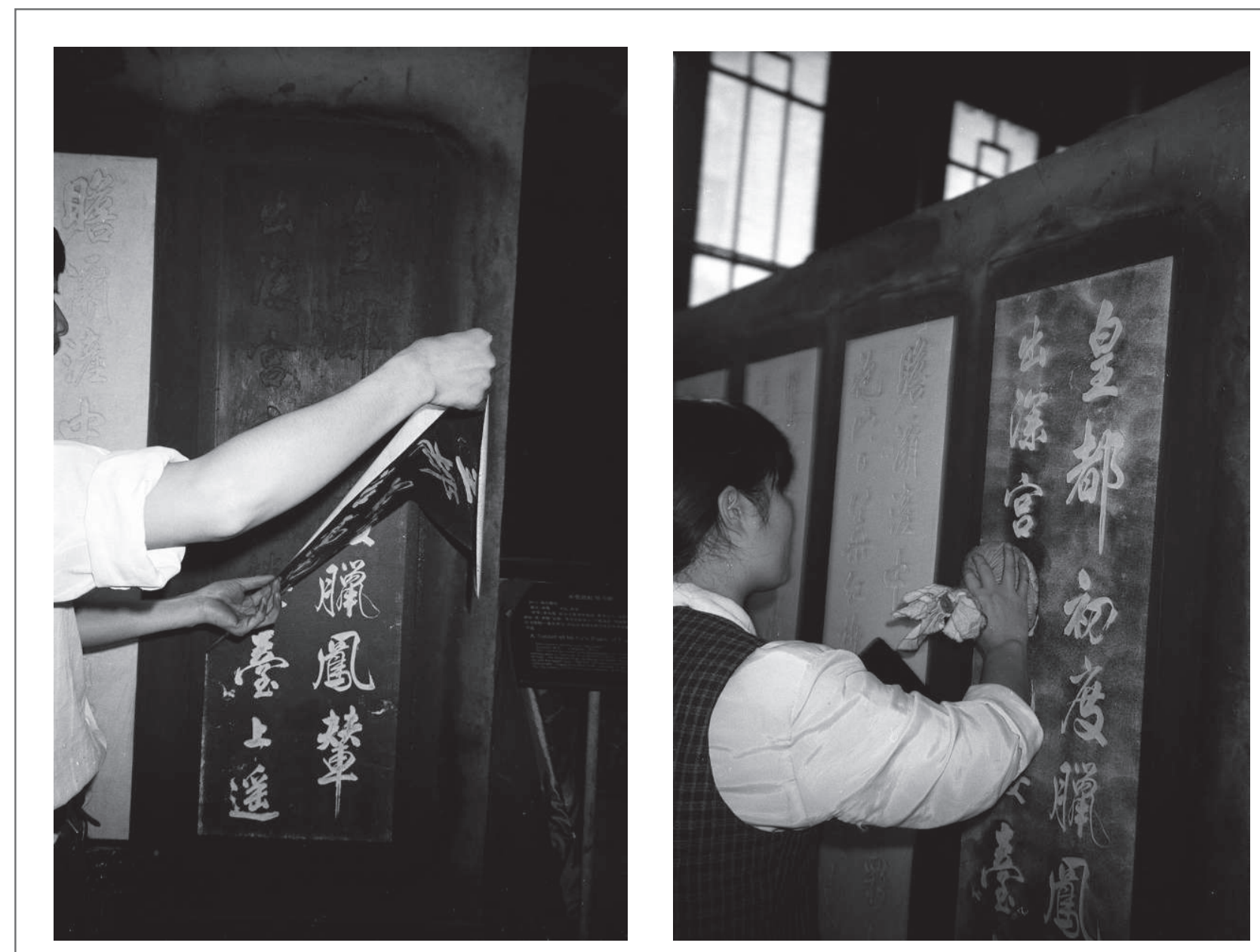

\section{Forêts de Stèles Xỉan, Chine, 1998}

${ }^{\circ}$ R. Cresswell

Dans une écriture idéographique chaque signe doit être la réplique parfaite d'un modèle standard. De la forme même de la communication, donc, dépend l'exactitude de la transmission du contenu. Gravés dans un matériau durable, les caractères normatifs sont décalqués par un typographe.

de «l'ambiance sociale » dans laquelle se nouent les interactions sociales. Les formes et les modalités de leur influence sont à découvrir dans nos recherches ultérieures, mais d'ores et déjà il est évident que seule une étude de structures peut répondre aux questions posées. Il faut analyser les rapports dialectiques entre techniques et culture, et la définition même de structure dit qu'elle est formée d'un ensemble de rapports. En face des structures sociales il faut constituer des structures techniques, point donc de typologie ou de technographie.

Le problème est vaste, car il met en relation non seulement les procédés techniques et les institutions sociales, ou plutôt la morphologie des institutions sociales, mais aussi l'homme et le milieu naturel par la médiation des techniques. La recherche nous amènera à nous interroger sur la nature des modèles et sur l'explication scientifique, sur la nature de l'invention technique et sur la constitution et l'interaction des ensembles techniques dans une même société, et enfin sur le possible caractère invariable, et sur la direction certaine, de l'évolution technique.

Malheureusement trop de travaux sur les techniques sont descriptifs, ne traitant que des objets et des outils, et non pas de leurs rapports structuraux. Or, l'objet ou l'outil ne nous intéresse qu'en ce qu'il constitue un chaînon dans un processus. 


\section{Épistémologie}

Un premier problème épistémologique est de bien expliciter ce qui est entendu par le terme: modèle. Le problème n'est pas, d'ailleurs, limité à notre recherche, il est au centre de tout effort scientifique actuel. Il est généralement admis qu'un modèle rend compte de la réalité en situant les éléments de cette réalité les uns par rapport aux autres, en un mot dans la structure. Il doit permettre en outre d'expliquer phénomènes observés, et de prédire les transformations ultérieures possibles. Il est moins généralement reconnu qu'un modèle, pour répondre à ces critères, doit rendre compte de toute la réalité à chaque niveau de son élaboration, et doit fonctionner de façon bilatérale, c'est-à-dire permettre le raisonnement du général au particulier aussi bien que celui du particulier en général. Cette nécessité élimine, il faut bien le dire, la plupart des prétendus modèles en ethnologie.

[... En première approximation nous pouvons souligner la différence entre un modèle scientifique (ex. la gravitation) et ce qui passe pour un modèle dans les « sciences » sociales, par exemple celui dit " analyse des commutations variantes " (cf. la parenté selon RadcliffeBrown). Dans celui-là il est possible sans intervention extérieure aussi bien d'induire le général à partir du particulier que de déduire le particulier à partir du général; dans celui-ci il est impossible de retrouver le particulier une fois que l'induction a abouti au général...]

\section{Problématique}

Le corpus des certitudes en sciences sociales n'est pas suffisamment riche pour livrer un ensemble de définitions dont on peut être sûr qu'elles resteront valables du début à la fin d'une recherche. Celles qui suivent servent de point de départ, mais il est plus que probable, il est même à souhaiter, que les termes auront à être affinés avant d'atteindre les conclusions. Commençons par la définition d'une technique. Celle-ci demande un agent, de la matière, et un instrument, liés dans un processus de transformation, ou encore un agent et un objet liés dans un processus de consommation.

Postulons ensuite que toutes les techniques peuvent être réparties en deux groupes: (1) les techniques qui expriment des rapports sociaux; (2) les techniques qui créent des rapports sociaux.

Dans le premier groupe il s'agit essentiellement de techniques de consommation, et il est probable que l'on puisse opérer une nouvelle division entre les techniques dont la connaissance diffère de groupe social à groupe social et celles dont l'instrument de travail ou l'objet de consommation expriment la différence sociale. Quant aux techniques qui créent des rapports sociaux, il semblerait qu'elles doivent être le sujet privilégié d'une nouvelle technologie, étant donné leur place prépondérante dans la société et, partant, leur importance pour l'analyse sociologique. Naturellement, des comportements ou des opérations relevant des deux groupes peuvent se trouver entremêlés dans un processus donné, mais le but principal (fabrication ou consommation de l'objet produit) permettra toujours de classer l'ensemble dans l'un ou dans l'autre groupe.

[...A. Leroi-Gourhan (ALG) classa les techniques selon les moyens d'action sur la matière, selon les méthodes de transformation de la matière, et selon les modes d'acquisition et de consommation des produits...]

Remarquons que nous touchons ici à une difficulté qui surgit souvent dans la sémantique de la technologie, voire dans celle de l'anthropologie globale: celle de la définition du 


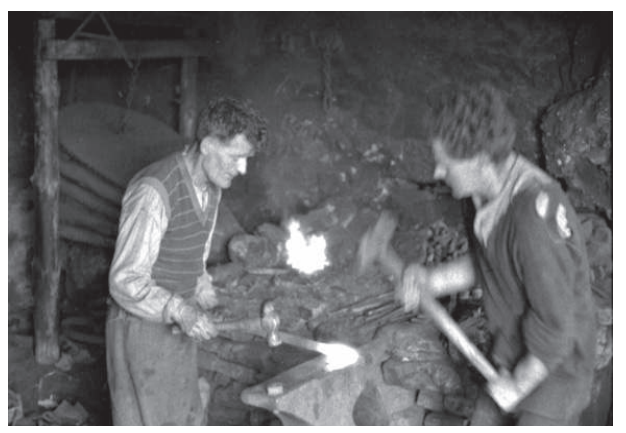

${ }^{\circledR}$ Kinvara Community Council Ltd - Archives R. Cresswell 1956 - 1957

Forgerons, Kinvara, Co. Galway, Irlande, 1956

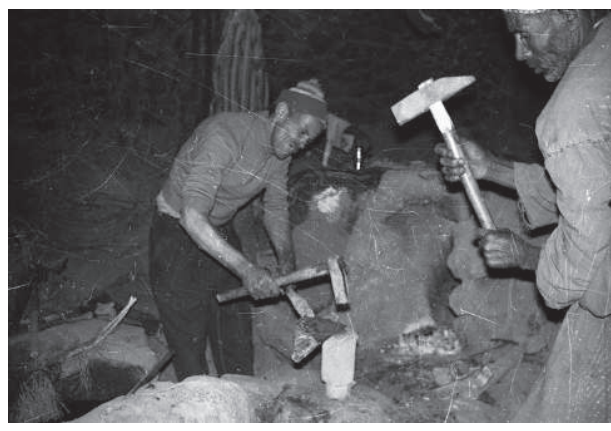

${ }^{\circ}$ R. Cresswell

\section{Forgerons, Ait Souka, Wilaya de Marrakech}

II semblerait que peu de différences existent entre la gestuelle technique d'un forgeron irlandais et celle d'un forgeron marocain. La suite technique : chauffe, frappe, trempe aurait raison des spécificités culturelles. Cette constatation met à l'épreuve une prémisse de ce programme de travail en technologie culturelle : un forgeron pratiquerait une gestuelle technique présentant plus de similarités aux gestuelles d'autres artisans de son groupe local qu'à celles des forgerons d'autres cultures. domaine que l'on se propose d'étudier. Nous la contournerons en rejetant les objets d'étude et en constituant des sujets d'étude. L'objet maison, par exemple, résiste au classement; le sujet construction englobe la technique dont le produit est la maison, de même que le sujet consommation englobe la technique de l'emploi de l'espace dont le cadre est la maison.

La discussion sur la transformation nous mène à une autre définition à préciser: celle de chaîne opératoire. Une chaîne opératoire est une série d'opérations qui transforment une matière première en un produit, que celui-ci soit objet de consommation ou outil. Cette série est constituée par un certain nombre d'étapes, chaque étape étant caractérisée par: un terme indigène, un terme « scientifique » (il peut arriver souvent qu'un seul nom vernaculaire traduise deux opérations techniques), un lieu, un outil, un geste, un type de percussion, une force, une matière première, un produit, un agent et un état de la matière (chimique ou physique...).

Chaque processus de fabrication peut mettre en jeu une ou plusieurs chaînes opératoires, et ces chaînes peuvent être intégrées de différentes façons.

\section{[...Définitions d'ensemble technique et d'unité de production...]}

\section{Méthodologie}

La discussion de la problématique et de l'épistémologie mène forcément à la méthodologie. Étant donné la nouveauté de ces recherches, les détails de la méthodologie seront à inventer au fur et à mesure du déroulement du travail, mais nous pouvons ici en rappeler les sources principales. Celles-ci sont au nombre de trois, les travaux d'André Leroi-Gourhan pour la description et l'analyse des techniques, les études de Claude Lévi-Strauss pour le codage et l'analyse des phénomènes socio-culturels, enfin les analyses socio-économiques de l'école marxiste pour la mise en place du cadre économique.

Leroi-Gourhan nous donne un cadre d'analyse et de description où ce sont les données intrinsèques des outils et des techniques qui fournissent les critères de la systématisation.

[...Classer les outils d'après le but poursuivi rend les classements tributaires de la façon dont les usagers les perçoivent, ce qui varie forcément selon l'époque historique. Les taxinomies d'ALG rangent dans une même classe les objets qui partagent les mêmes traits mécaniques et physiques. Le cadre général de ses travaux se dresse selon la notion de tendance et fait. La tendance rend compte des séries logiques ahistoriques, les faits sont rangés en des degrés de plus en plus précis pour aboutir à un outil ou un produit chez un groupe social réel dans un lieu géographique nommé à une époque datée. Cet ordonnancement des gestes techniques permet à la fois de situer la logique solidement dans l'histoire, et d'ouvrir une porte théorique à la réalité matérielle...]

Il faudrait aussi faire appel aux classements indigènes, d'autant plus que nos catégories pousser, tirer, taper, écraser... ne correspondent pas toujours aux seuls critères internes. 


\section{$[\ldots]$}

[Que] les Papago classent ensemble dans leur taxonomie poule et chien, porc-épic et tortue (Pilcher, 1967), ne relève ni d'une fantaisie quelconque, ni d'une incompréhension du monde naturel, mais simplement de ce que leur taxonomie a une orientation différente de celle que nous appelons scientifique. Une taxonomie doit en principe éviter de classer un même objet dans deux catégories différentes, mais une hiérarchie des critères est déterminée socialement. Si [la] taxonomie est à orientation scientifique (mode de connaissance) la hiérarchie est plus simple à chaque étage et va du particulier

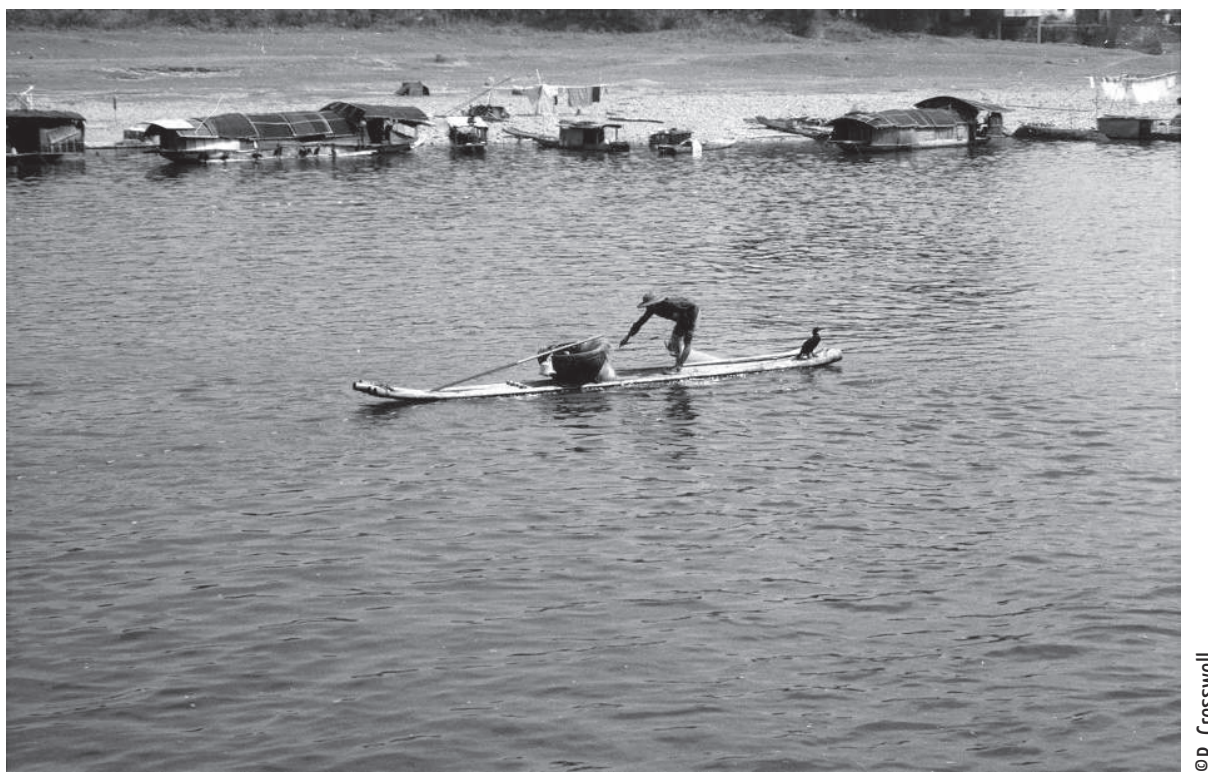

\section{Radeau de pêche au cormoran sur le Li entre Guilin et Yangshuo, Chine}

Cette activité techno-économique était autrefois très répandue dans la région, mais aujourd'hui elle est surtout réduite au secteur du tourisme. Ici les exigences de la fonction technique sont peu importantes, et cela se reflète dans la simplicité de la forme. Un volume suffisant de bois pour supporter un pêcheur, un panier pour contenir les résultats de la pêche, une lampe pour attirer les poissons (il s'agit essentiellement de pêche nocturne), et un cormoran, le tour est joué.

au général. Mais il est tout aussi valable de créer une

taxonomie à orientation technique (mode d'utilisation) où la hiérarchie est déterminée par le but social. Les Papago sont parfaitement cohérents lorsqu'ils placent ensemble poule et chien qui sont des êtres vivants qui bougent, qui n'ont pas peur de l'homme et qui sont la propriété des membres de la société. On peut sans trop de difficulté imaginer qu'à l'aide de tels classements les ensembles techniques d'une population puissent être étudiés de façon fructueuse.

L'emploi de ces concepts et de ce type d'analyse doit aboutir à la structuration des techniques. Si l'on veut déterminer les rapports entre structures techniques et phénomènes socio-culturels il faut évidemment connaître les structures de ceux-ci. Pour cette raison [...je propose que l’on s'inspire...] des méthodes d'analyse structurale développées par Lévi-Strauss. Il ne s'agit pas en l'occurrence d'un éclectisme qui chercherait à réunir en une seule méthodologie deux chefs de file mais simplement d'une recherche qui nécessite l'emploi des meilleurs outils conceptuels disponibles.

Pourquoi le structuralisme? Parce que l'analyse structurale à l'heure actuelle est [...] à même de codifier et la parenté et les systèmes symboliques des sociétés à étudier, non pas uniquement en termes d'éléments constitutifs, mais surtout en termes de rapports entre ces éléments. Les modèles construits ainsi rendent compte non seulement des structures profondes cachées par le caractère infiniment varié et unique des actes de la vie quotidienne, mais aussi des transformations et des opérateurs de transformations propres à ces structures. En analysant à la fois les réalités abstraites qui sous-tendent les réalités 
tangibles, et l'aspect dynamique de ces mêmes réalités, la recherche structuraliste atteint les charnières par où agissent les forces d'évolution, et, de ce fait, présente un modèle qui peut être mis en parallèle avec les modèles des forces productives et techniques. De plus on ne peut trop souligner que ce genre de recherche livre une construction faite de rapports évitant ainsi le piège des listes stériles de traits.

À cet égard, il s'agira de tenir compte non pas d'un seul mais de plusieurs niveaux d'analyse. La plupart des structuralistes pensent que le premier niveau d'abstraction atteint déjà les structures profondes. Une recherche concernant la structure dans certaines sociétés du Moyen-Orient semble démonter au contraire qu'entre le niveau des comportements uniques et des institutions particulières et celui des structures profondes existe un autre niveau où le concept même de structure est spécifique à chaque culture (Cresswell, 1966). [...] Dans ce cas il est certain que les premiers travaux sur les rapports entre techniques et manifestations culturelles doivent se situer à ce niveau avant de tenter l'élaboration d'hypothèses valables pour toutes les cultures.

Il ne suffit pas de mettre en place deux structures, fussent-elles élaborées dans le cadre d'une même épistémologie, pour que les rapports et les influences dialectiques que nous recherchons deviennent immédiatement visibles. Un cadre théorique général est nécessaire, et se trouve dans les travaux de l'école marxiste, la troisième source d'une technologie nouvelle.

Il y a plusieurs raisons d'utiliser ces catégories, et cette méthodologie. Tout d'abord remarquons que l'analyse anthropologique, parce que dans un certain sens elle explore dans le détail une très grande aire d'activités humaines (pratiques culinaires, gestuelles, rituelles, etc.), utilise deux catégories séparées (techno-économique et manifestations socioculturelles) concernant des éléments différents pour les besoins son analyse.

[... cf. supra pour les taxinomies d'ALG. L'intérêt du mode de production comme concept d'étude est qu'il propose d'utiliser les mêmes termes, linguistiques ou autres, pour traduire à la fois les aspects techno-économiques et sociologiques d’un phénomène de société... .]

Deux écueils principaux sont à éviter: des hypothèses mécanistes, une recherche réductionniste. Aux critiques d'un prétendu aspect mécaniste de ces recherches à ceux qui priment le discours verbal, dont, pourtant, la transmission d'un chercheur à un autre est combien difficile, il est possible de répondre, entre autres, que loin de négliger les valeurs attachées aux comportements et les connaissances qui les précèdent, valeurs et connaissances doivent s'intégrer dans l'analyse. Il ne s'agit pas de déduire les formes d'organisation sociale à partir de la forme d'un bâton à fouir, mais, au contraire, de montrer comment le bâton à fouir s'intègre dans un processus de travail, lui-même s'intégrant dans l'ensemble des comportements sociaux de la société globale.

Quant à la critique éventuelle de réductionnisme, ce qui a déjà été dit sur la nécessité de garder toute la chair de la réalité au moment de l'élaboration de modèles scientifiques semble une réponse satisfaisante pour l'instant.

[...Une généralisation qui soustrait des éléments importants d'analyse à chaque niveau d'abstraction est critiquable...] 


\section{Évolution technique}

[...]

L'évolution humaine est un fait indéniable, ou, si le mot évolution porte trop la nuance d'un jugement de valeur, le changement survenu depuis l'aube de la race humaine est un fait indéniable. Il y a [plusieurs] million[s] d'années nos ancêtres étaient des chasseurscollecteurs des forêts et des savanes, aujourd'hui nous explorons l'espace. Que cette transformation de nos possibilités soit due à une maîtrise technique n'est pas moins évidente. Ce fait permet et a permis de dresser une sorte de tableau où l'on voit les groupes humains passer de la chasse et de la cueillette à l'élevage et l'agriculture avant d'élaborer une société industrielle. On peut même affiner ce tableau comme l'on voit dans la figure 1 qui suit, où paraissent les deux grands bonds en avant de l'humanité, la révolution néolithique et la révolution industrielle. Il est même possible de préconiser un autre grand bond qu'aurait été l'invention de l'outil. Dans le même tableau se voient également le développement de l'établissement humain, passant d'un état nomade à un état sédentaire, et, à partir de la révolution industrielle, la scission en deux populations : les citadins et les paysans. L'économie de subsistance est devenue une économie de surplus, et celle-ci a débouché sur ce qu'il est convenu d'appeler une « civilisation des loisirs ${ }^{1} »$. Enfin, avant la révolution néolithique nous vivions dans un écosystème naturel, puis nous sommes passés à un écosystème agricole, et aujourd'hui il nous est possible de fabriquer un micro-milieu parfaitement artificiel et d'y subsister.

Séries logiques de développement technique.

(Fig. 1)

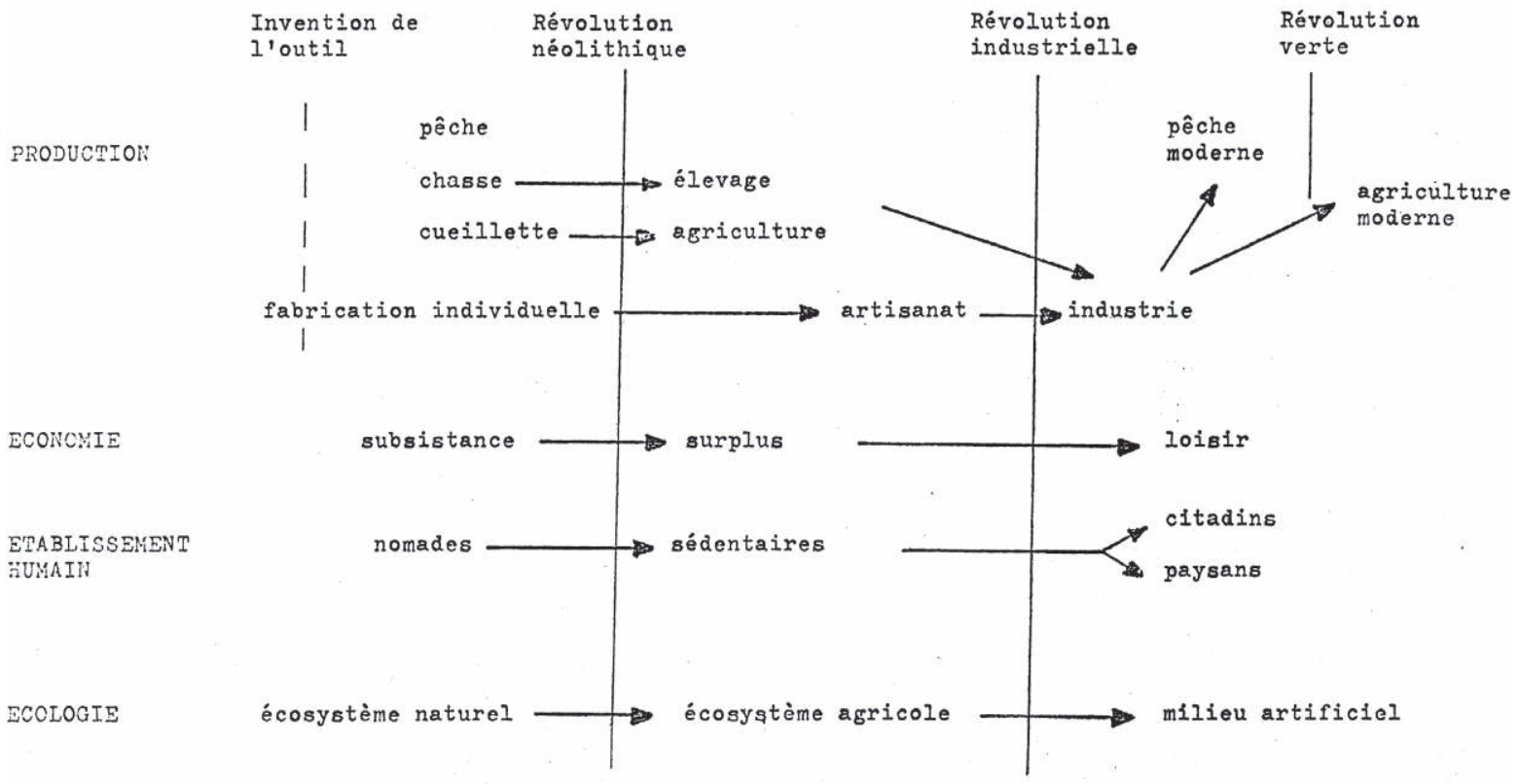




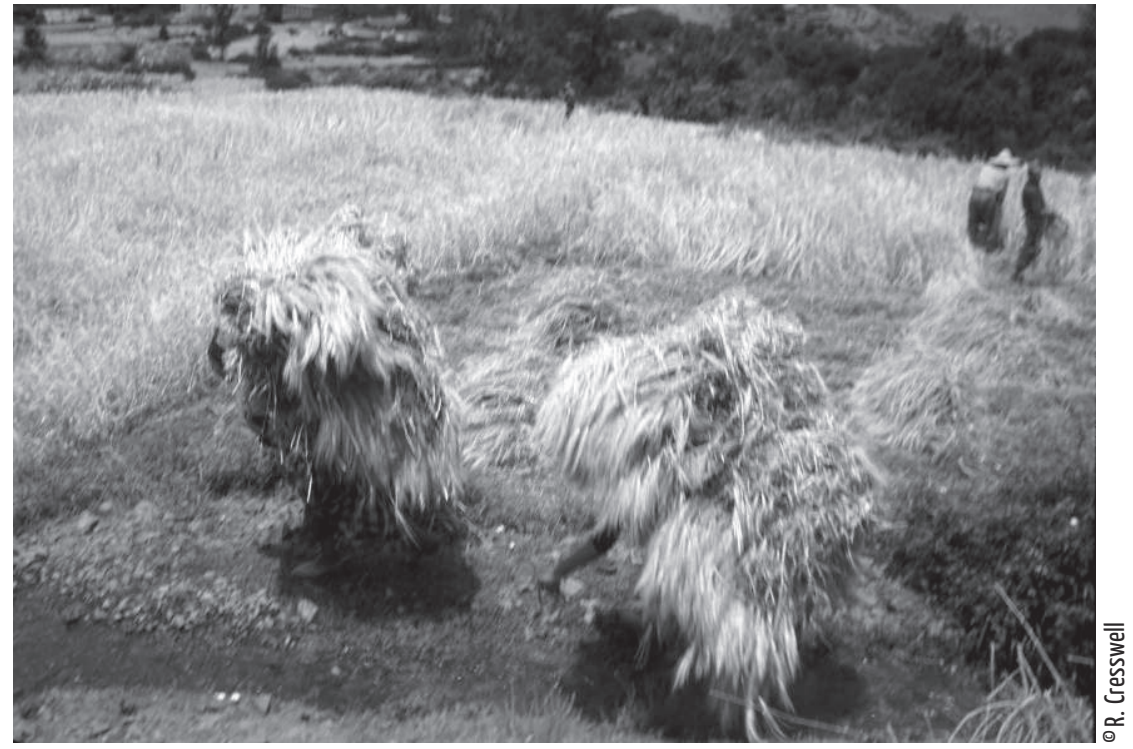

Marocaines transportant des bottes de la récolte à la maison, Imlil, Wilaya de Marrakech, 1981

II est nécessaire de bien distinguer dans l'analyse de chaînes opératoires les critères qui partagent le travail entre hommes et femmes. II ne s'agit manifestement pas de force physique.
Apparemment ce tableau est à la fois logique - l'évolution technique est linéaire et va du simple au complexe, comme il se doit - et historique - les différents peuples du monde étant passés par ces stades à des moments différents de leurs histoires. La lecture de ce tableau suscite encore d'autres commentaires: par exemple, l'état chasseur-cueilleur est lié à un mode de vie nomade et à une économie de subsistance. Ou encore le passage d'une économie de subsistance à une économie de surplus représente le passage d'une économie irrationnelle à une économie rationnelle. Ou encore, au niveau des techniques

de transformation, le surplus dans une société permet de passer de la fabrication individuelle à la fabrication artisanale et à la création de catégories de fabrication spécialisées, nourrit par les travaux de ceux à qui ils fournissent les outils. Ou enfin que la révolution néolithique est un phénomène du même ordre que la révolution industrielle ou la révolution verte.

Il va sans dire que si ces différentes remarques ont été signalées c'est qu'elles sont toutes fausses.

Prenons les points un par un. Les chasseurs-cueilleurs sont des nomades, c'est l'invention de l'agriculture qui permet la sédentarisation et la création de réelles agglomérations. Du Croissant Fertile vient la négation de cette idée reçue.

[... Suberde, 6500 av. J.-C., en Turquie méridionale, regroupait une population de chasseurs sédentaires. Mallaha, 9000 av. J.-C., en Palestine, était un village de chasseurs-cueilleurs à maisons en pierre. Enfin, les Kwakiutl des côtes du Pacifique canadien donnent l'exemple d'un groupe à production alimentaire rustique et à développement sociétal hautement complexe...]

L'économie de subsistance est souvent irrationnelle. On évoque pour étayer cet énoncé le cas de certains peuples d'Océanie qui procèdent périodiquement à des grands massacres de porcs.

[... Les exemples trouvés dans la littérature citent souvent comme preuve de pensée économique irrationnelle les grands sacrifices d'animaux ou la destruction de produits végétaux, voire d'objets matériels - les Tsembaga en Mélanesie, ou encore les Kwakiutl. L'analyse démontre que ce comportement correspond surtout à des contraintes écologiques...]

La thèse classique concernant la notion de surplus dit qu'avec l'invention de l'agriculture et l'augmentation de la production qui s'en est suivie, et avec la possibilité de stocker la nourriture, donc d'assurer une distribution annuelle égale, des groupes de spécialistes se sont constitués, échangeant leurs services ou leurs objets fabriqués contre la nourriture produite par les agriculteurs. Ainsi se seraient formés les artisans, les guerriers, 
les prêtres, les administrateurs, ainsi seraient nées les cités et les institutions étatiques. Implicitement cette théorie dit que là où existe le surplus se formeront les groupes spécialisés et apparaîtront les institutions complexes.

L'examen d'un groupe comme les !kung, Bochiman de l'Afrique du Sud, fait lever un premier doute sur la justesse de cette thèse (Lee, 1969).

[...65\% des !kung ne travaillent qu’un jour sur trois, mais vivent avec un régime alimentaire de 2200 kilocalories et 93 grammes de protéines par jour. L'idée qu’un surplus est le seul facteur à l'origine des grands États paraît suspecte...]

Remarquons, d'ailleurs, que la notion même de surplus est une notion ambiguë; entre autres s'agit-il de ce que les gens ne consomment pas ou s'agit-il de ce que les gens ne peuvent pas consommer, car repus. Pourtant il est évident que les non producteurs doivent échanger leur travail ou leur service contre de la nourriture. Pour résoudre la difficulté il semble raisonnable d'émettre l'hypothèse que le surplus n'est jamais absolu, et que donc dans ces premières civilisations il n'existait pas d'abord un excédent de production et qu'ensuite des groupes non producteurs se sont constitués. Tout porte à croire que le surplus utilisé à nourrir les non producteurs était un surplus artificiel créé en soustrayant de la production globale d'un groupe une certaine quantité de produits alimentaires, réservés alors aux artisans et aux chefs politiques et religieux. L'invention et le développement de l'agriculture auraient été donc un effort pour combler un déficit de production crée par la constitution d'un surplus artificiel.

Examinons enfin l'idée que la révolution néolithique, l'invention de l'agriculture, est un phénomène de même ordre que la révolution industrielle.

[... Les fouilles minutieuses à Tehuacan, vallée au sud-est de Mexico, fournissent des témoins matériels de la suite des étapes du développement d'une agriculture spécifique, depuis les quelques pieds plantés devant les cavernes (10700 av. J.-C.) jusqu'à la manipulation génétique du maïs (1500 apr. J.-C.)...]

Retenons de l'exemple de Tehuacan deux points. [Première constatation: les étapes agricoles se suivent dans un ordre logique] La deuxième constatation est la lenteur de cette évolution. Pendant plus de mille ans le maïs sauvage constitue $93 \%$ de tout le maïs consommé. Il se passera encore 1200 ans avant qu'il y ait plus de maìs domestiqué dans le régime alimentaire que de maïs sauvage et encore 2700 ans avant que les maïs hybrides dépassent $51 \%$ de la nourriture. Et 1000 ans sépareront les premiers efforts de réelle domestication attestée par la présence des maïs hybrides, et l'apparition de villages fixes sur le territoire. Révolution semble un terme tout à fait impropre pour décrire des périodes de cet ordre de grandeur.

Revenons au tableau. Bien qu'il faille rejeter un certain nombre d'idées reçues dans chacune des progressions (l'irrationalité de l'économie, la notion de surplus, la vitesse de la révolution néolithique), l'ordre des étapes dans chaque domaine semble résister à la critique. Par contre la correspondance entre les différents étages de l'ensemble s'écroule irrémédiablement. Lagriculture peut s’accompagner de la chasse, ou se passer d'activités cynégétiques, comme à Tehuacan. Les chasseurs-cueilleurs peuvent être sédentaires. L’agriculture à ses débuts se traduit par une économie de subsistance. L'élevage même peut se passer dans un écosystème naturel.

Posons alors comme hypothèses les énoncés qui suivent. Il existe une progression réelle dans les techniques, il s'agit donc d'une série logique. Mais l'ensemble représenté sur le tableau n'est pas un ensemble monolithique. Chaque série (la pêche évoluant vers une pisciculture, la chasse vers l'élevage, une économie de subsistance vers une économie de surplus et de marché) possède une certaine autonomie propre et la forme particulière d'imbrication de ces 
séries spécifique à chaque société, représente l'histoire. Peut-on parler alors d'une évolution historique? Schématiquement il semble préférable de conceptualiser la progression historique comme une série d'étapes discrètes d'adaptation sociale à un substrat techno-économique, qui lui-même possède la potentialité de progression.

\section{Les techniques qui créent les rapports sociaux}

\section{L'étrier}

La littérature sur les effets provoqués dans la société par un changement technique est abondante, mais personne ne l'a traitée avec plus de maîtrise ou plus clairement que Lynn White dans son livre sur les techniques médiévales et le changement social (1962). [...L'importance soudainement donnée à la cavalerie, le changement des dates des grandes réunions des militaires, la transformation du langage de l'équitation sont des témoins d'une invention de portée profonde. Pour l'auteur il s'agit de l'introduction de l'étrier qui au moyen d'une meilleure assise du cavalier permit la constitution d'une nouvelle cavalerie. La barre transversale derrière la tête de lance qui apparaît à la même époque montre que cette cavalerie était lourde...]

Les effets de l'introduction de l'étrier dans la société carolingienne furent multiples. À part la création du cadre foncier et social de la féodalité médiévale, le développement de l'armure, des armes, d'une race de chevaux puissante, surtout dans cette économie d'échanges en nature, qui était celle des viII et $\mathrm{IX}^{\mathrm{e}}$ siècles, aboutit à la création d'une catégorie sociale nouvelle dans l'Europe de cette époque: le guerrier professionnel. Lapparition de ces guerriers et leur fixation en quelque sorte sur le sol contribuèrent à l'élaboration de tout un nouveau système de statuts et de rôles. Mais l'arrivée de l'étrier ne précipita pas automatiquement tous ces changements, il fallait au préalable reconnaître les avantages qu'il était potentiellement en mesure de conférer. Les Francs les reconnurent, les Saxons y furent aveugles, et quelques siècles plus tard les Normands, héritiers des Francs et de leur façon de guerroyer, battirent les Saxons à Hastings, grâce aux cavaliers lourds.

\section{Charrue, collier d'épaule, assolement triennal}

La première synthèse explicative de l'introduction de la charrue en Europe a été proposée par Marc Bloch. À l'origine les instruments aratoires étaient des araires, ce qui nécessite un labour croisé, et la forme carrée des champs en résulte.

[... Les sols lourds du nord de l'Europe sont mieux préparés à la culture avec une charrue munie d'un coutre et d'un versoir...]

La charrue contribua puissamment au développement du système seigneurial, car, exigeant des attelages de plusieurs paires de bøufs, plus que ne pouvait entretenir un seul exploitant, les équipes de travail qui se constituèrent en conséquence provoquèrent la croissance des groupements d'agriculteurs. Pendant la même période les chevaux se 
substituèrent aux bœufs comme animaux de trait. Ce remplacement tient à deux inventions premièrement un harnais qui n'étouffe pas le cheval pendant son effort de traction, deuxièmement l'introduction vers le ix $x^{e}$ siècle du fer à cheval.

[... Le grand avantage de vitesse du chevalin par rapport au bovin ne pouvait jouer avant l'introduction du collier de cheval, car le joug sur un cheval comprimait la respiration de l'animal lors de la traction, là où le collier portait l'effort sur l'épaule. Cette théorie de Lefebvre des Noëttes doit probablement être réajustée pour tenir compte de la taille de l'animal et des points de traction du chargement tiré. Par ailleurs le labourage sans croisement mène aux champs en lanières, donc aux « openfields » qui nécessitent des conseils villageois...]

L'avantage du changement [d'assolement] ${ }^{2}$ est réel. Si l'on prend à titre d'exemple une communauté avec 600 ha de terres arables et qui a l'habitude de labourer deux fois sa jachère un simple calcul montre que dans un assolement biennal, 900 ha doivent être labourés pour 300 ha de plantés. Si l'assolement passe de biennal en triennal la communauté aura à labourer une sole de 200 ha une deuxième sole de 200 ha et une troisième sole en jachère labourée deux fois, ce qui lui fait en tout 800 ha de labour pour 400 ha de plantés. Mais elle avait auparavant la possibilité de faire 900 ha de labours, elle peut donc redistribuer les 100 ha en excédent par un défrichement de 25 ha par sole; ce qui lui fait 225 ha à labourer dans une sole, 225 ha dans une autre, plus 450 ha de labours pour la jachère, soit 900 ha de travail pour 450 ha cultivés un gain de 50 \% dans sa production.

\section{L'invention}

L'introduction donc de nouvelles techniques dans la société fait ressortir un grand ensemble de facteurs qui s'imbriquent dans un jeu compliqué. Retenons pour l'instant deux notions qui paraissent fondamentales celle de terrain favorable à l'invention - l'étrier n'a pas été un précurseur de la féodalité partout -, et celle de la dialectique entre les rapports techniques et les rapports sociaux de production.

Une autre notion qui paraît fournir l'une des clés pour la compréhension de l'invention est celle de progression technique par paliers. Par cela s'entend que le progrès technique n'est pas une fonction linéaire, mais exponentielle. Par exemple, lorsqu'on examine les différentes presses utilisées dans le bassin méditerranéen pour exprimer l'huile d’une pâte d'olives broyées, on trouve pour la plupart des presses traditionnelles une pression qui varie entre $2 \mathrm{~kg}$ par $\mathrm{cm}^{2}$ et $6 \mathrm{~kg}$ par $\mathrm{cm}^{2}$, et cela pour exprimer environ $18 \mathrm{~kg}$ d'huile d'une pâte pesant $100 \mathrm{~kg}$ (Cresswell, 1965). Lorsqu'on projette ces données sur un graphique cartésien, en y incorporant le temps de la presse, et que sur ce même graphique on projette les données des presses à olives modernes, hydrauliques, on s'aperçoit qu'il ne suffit pas d'augmenter la pression de quelques $\mathrm{kg} / \mathrm{cm}^{2}$ pour obtenir davantage d'huile. Il faut une force supérieure de sept à dix fois pour un ou deux kilogrammes de plus d'huile. Le trait qui relie les deux constellations de points sur le graphique est donc une courbe exponentielle, l'interprétation de ce phénomène - et on le retrouve dans bien d'autres cas techniques - semble être que, dépassé un certain niveau de perfectionnement, le progrès technique est qualitatif et non plus simplement quantitatif.

Avant de passer aux techniques qui créent des rapports sociaux, posons une des questions auxquelles les travaux sur les liens entre phénomènes techniques et manifestations socio-culturelles devraient être en mesure de répondre à la fin des recherches: pourquoi 
la révolution industrielle n'est-elle pas née en Chine? Lorsqu'on observe une liste des inventions qui ont joué le plus grand rôle dans le développement technique et partant, social de l'Occident, il est étonnant de constater que la plupart sont arrivées d'Asie, surtout de Chine. Entre le iv et le ${ }^{e}$ siècles, pour ne citer que les plus importantes, l'Occident a emprunté à la Chine le métier à tisser à navette, l'étrier, le cardan, le collier de cheval, et le trébuchet. Ensuite, du XI ${ }^{\mathrm{e}}$ au xiII ${ }^{\mathrm{e}}$ siècles, nous remarquons le zéro et la valeur de position en mathématiques, la boussole et le gouvernail, le papier, la brouette et le moulin à vent. Entre le xiII et le XviII ${ }^{\mathrm{e}}$ siècles, les Chinois nous ont livré l'horloge mécanique, le fourneau de fonderie, l'imprimerie par blocs, le volant à chaîne et boules (si important pour l'invention de la machine à vapeur), les portes d'écluse, le pont à suspension, les compartiments étanches de bateaux, etc. Alors comment se fait-il que la révolution industrielle soit occidentale et non point chinoise?

Les chercheurs qui se sont déjà attaqués à ce problème développent l'argument suivant. La Chine depuis très longtemps était un État centralisé dont le pouvoir central était en rapport direct avec les groupes locaux et s'appuyait sur une armée de fonctionnaires Il n'y avait donc pas d'instances politiques intermédiaires plus ou moins indépendantes favorisant le développement, comme c'était le cas en Occident, d'une classe de marchands et la constitution du capital. Sans classe capitaliste, la révolution industrielle ne pouvait avoir lieu. Wittfogel (1964) ajoute que la raison de la création d'un pouvoir fort en Chine était la nécessité de gérer de vastes travaux hydrauliques nécessaires à la production chinoise.

Mais un autre aspect du problème me paraît également demander droit de cité. Si le capital était effectivement fondamental pour la transformation de l'artisanat en industrie en Europe, tous les chercheurs s'accordent à dire que la pensée scientifique n'en était pas moins nécessaire. Nous pouvons donc nous demander si cette pensée scientifique européenne ne naquit pas elle-même des structures foncières et sociales de la féodalité. En effet la hiérarchisation et l'abstraction si nécessaires au développement d'une pensée scientifique semblent refléter fidèlement la structure sociale féodale qui, partant du serf, passe par les vassaux pour aboutir à un suzerain, clé de voûte de tout le système. Or, cette structure sociale féodale était elle-même, en partie tout au moins, fonction du développement technique des sociétés qui composaient l'Occident d'alors.

L'état des techniques chinoises contribua au développement d'une certaine forme d'organisation sociale, dont l'un des aspects était l'absence d'un capital marchand et de ce fait de l'une des bases de la transformation industrielle. À l'autre extrémité du continent eurasiatique, l'état des techniques européennes médiévales contribua au développement d'une forme d'organisation sociale qui, elle, inscrite sur le sol, favorisa l'éclosion de la pensée scientifique moderne. Pour développer ces hypothèses la recherche aura à résoudre, entre autres, le rapport entre invention technique et science. 


\section{Un exemple de technique qui reflète les rapports sociaux: l'habitation}

Il n'est pas question, bien entendu, d'aborder une étude en profondeur de l'habitation, il faudra se borner à définir le champ de l'étude et suggérer quelques méthodes d'approche. Au départ d'une analyse du cadre bâti des hommes, on ne peut manquer d'être appréhensif devant l'étendue de la tâche. Une multiplicité de formes d'assemblage de matériaux, une infinie variété des formes d'aménagement de l'espace, une distinction entre architecture vernaculaire et architecture savante, des maisons individuelles, des bâtiments publics, des usines et autres lieux de travail, des monuments, confondent celui qui voudrait relever des régularités, ou découvrir des déterminismes.

[...Appelons « habitation » les éléments du cadre bâti, et « habitat » ce qui est structure du même cadre...]

Dans la série logique de la maison il faut probablement distinguer entre ce qui est abri et ce qui constitue un espace différent. Une cabane gauloise de $3 \mathrm{~m}^{2}$ peut difficilement être autre chose qu'un abri-lieu de rangement ou cuisine, elle ne peut en aucun cas être le lieu où se passent toutes les activités domestiques de l'unité sociale fondamentale. Par contre, la clôture de l'espace où se passe une part importante des activités quotidiennes différencie ce qui est à l'intérieur de ce qui est à l'extérieur.

Examinons rapidement quelques méthodes d'approche du problème, et commençons par la forme extérieure de l'habitation souvent proposée comme critère de classement et d'analyse. L'examen d'une carte de l'Afrique occidentale, par exemple, comportant pour chaque groupe la description de la maison fait apparaître un chaos de formes variées: maisons rondes à murs d'argile et toit de chaume, maisons rectangulaires à murs en matières végétales et toit à deux pentes de chaume, maisons rectangulaires avec toit à quatre pentes, maisons entièrement en argile, maisons à terrasse, etc. Aucun regroupement ne se dessine, aucune régularité ne se dégage.

Pour classer les habitations recherchons [donc] une [autre] méthode d'analyse, [celle] qui tient compte non pas de la morphologie extérieure mais des principes de construction.

[... La figure 2 donne le détail d’un tel classement. L'emploi de celui-ci aboutit à doter chaque construction d'un indice...]

Plaçons sur la carte de l'Afrique ces indices à la place des dessins des formes de construction et nous voyons immédiatement ressortir les grandes zones naturelles de la région. Nous avons en réalité dessiné les contraintes et les possibilités techniques offertes par le milieu naturel. La fantaisie et les contraintes culturelles des groupes humains peuvent maintenant se donner libre cours à travers la forme que prendront les différentes constructions. Classer les formes, donc, revient non pas à classer des techniques de construction mais à classer des symbolismes culturels. L'étude de la forme d'une maison renseigne sur son origine culturelle, mais elle laisse très peu d'indications sur sa place dans les structures techniques. On connaît, d'ailleurs, en Afrique le cas de peuples descendus des montagnes dans la plaine, gardant la même forme de maison, mais obligés de transformer leurs procédés de construction.

L'étude de la forme pure lorsqu'il s'agit des plans mène vers le même type d'impasse. Si l'on regarde le plan d'une construction comme Pueblo Bonito au Nouveau Mexique (USA) (Fig. 3a), il est facile de trouver des analogies avec les immeubles d'appartements 
Matériaux

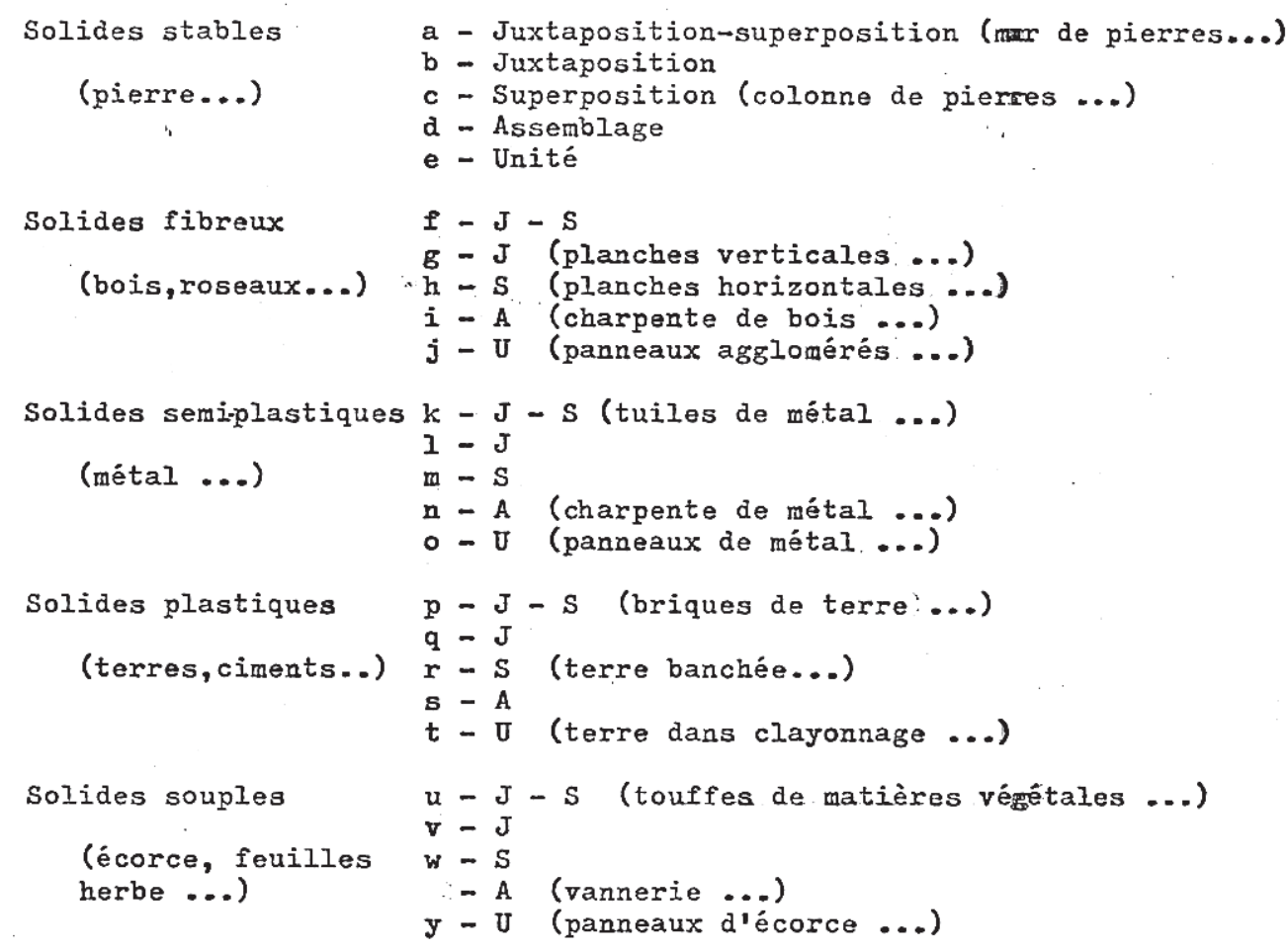

\section{Formes}

A. Simples

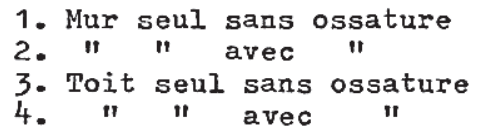

B. Murs porteurs

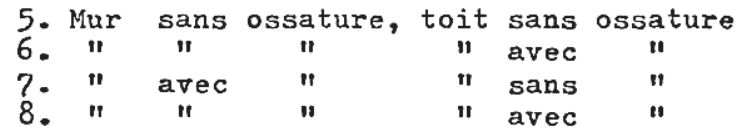

C.' ossature

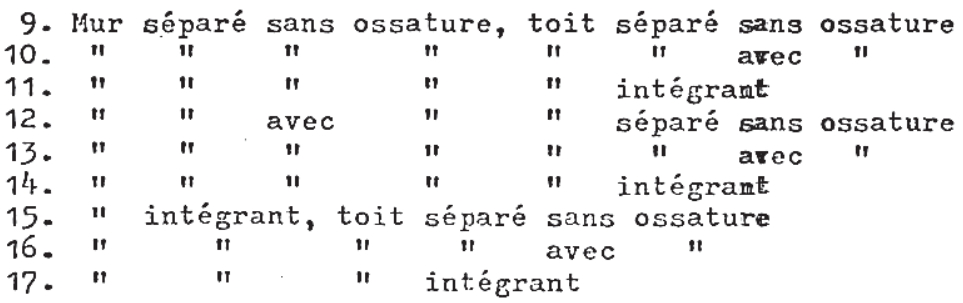

Classement des habitations Matériaux et formes.

(Fig. 2) 


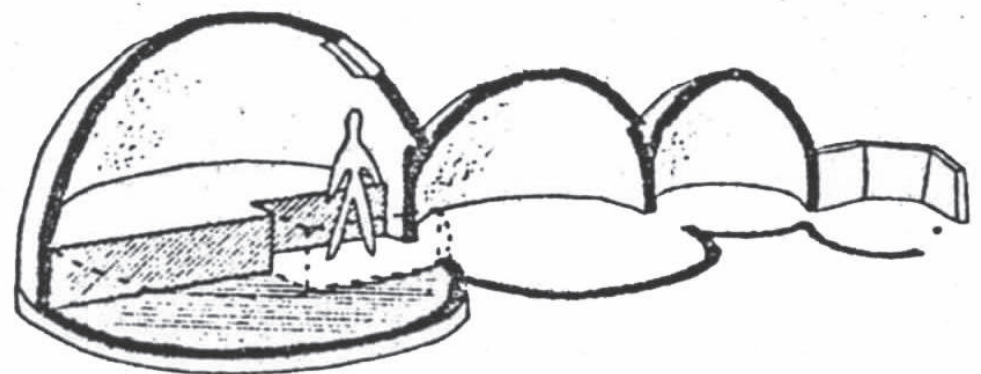

Esquimaux, Canada

Forme simple, un genre de toit posé sur le sol,

(d'après T. Mathiassen, 1928).
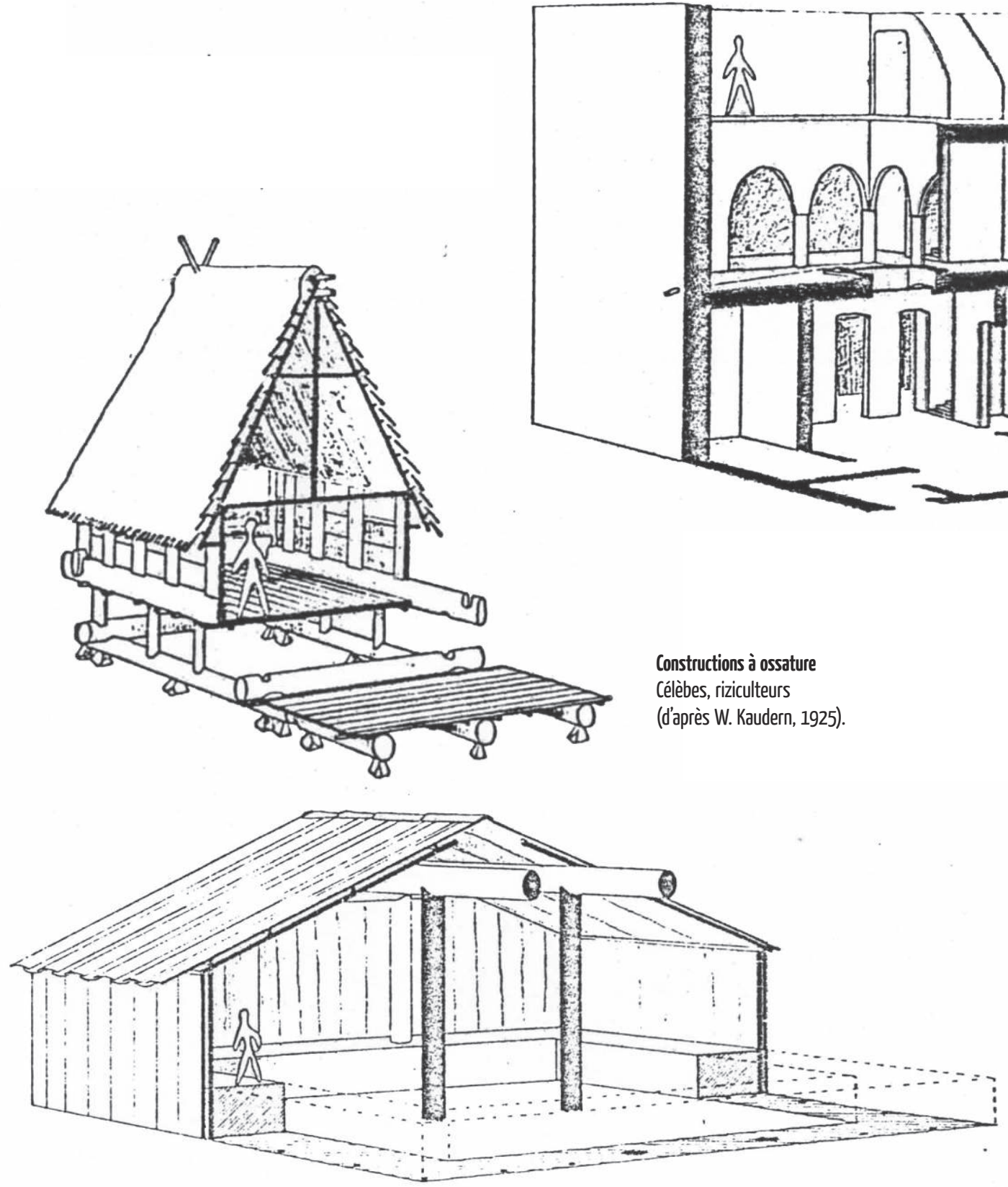

Constructions à ossature

Kwakiutl, Colombie

(d’après F. Boas, 1899). 
des villes industrielles modernes. On y voit des pièces allouées aux familles, des places ouvertes de réunion, des salles de réunion privées (kiva). Poser le plan de la longue maison des Dyak de Bornéo en regard (Fig. 3b), c'est appeler la même analogie - immeuble d'appartements - en remarquant que la forme diffère. Un examen plus approfondi des deux plans fait pourtant apparaître qu'ils sont de nature très différente.

Type de plan Tendance « cellulaire ». Pueblo Bonito, États-Unis

(d'après N. Judd, 1904).

Les pièces circulaires sont des kiva.

(Fig. 3a)

Type de plan

Tendance « unitaire ». Dyak, Bornéo

(d'après D. Freeman, 1955).

1. chambre de famille,

2. couloir,

3. emplacement réservé à cette famille,

4. véranda.

(Fig. 3b)
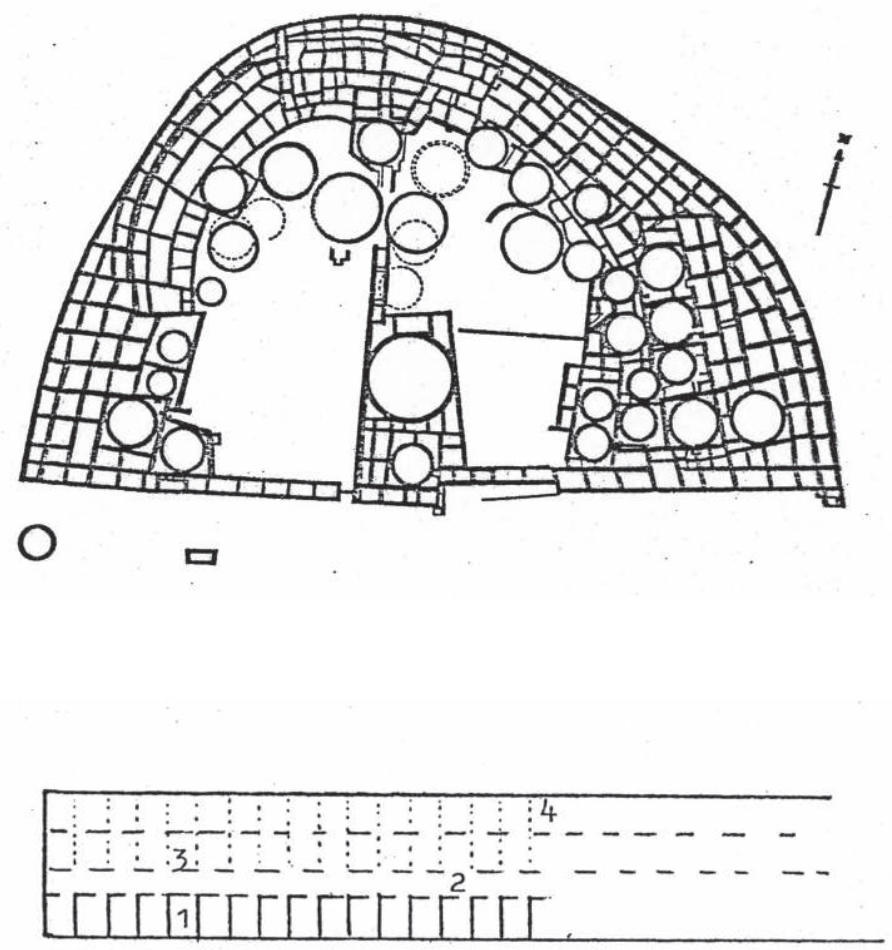

$1 / 1350$

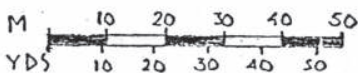

Examinons d'abord Pueblo Bonito. Il faut savoir qu'il s'agit d'une construction étagée, le mur nord comportant jusqu'à quatre planchers. Des quelque 800 pièces, donc, plus de la moitié se trouvent ainsi privées de lumière. De plus l'exiguiité des chambres mène à la conclusion (étayée il est vrai par l'observation des activités quotidiennes des Pueblo actuels) que la plupart des travaux domestiques se passaient à l'extérieur sur les terrasses et places. Donc, malgré une certaine désintégration de la vie communautaire, désintégration traduite par la multiplication des kiva à rôle socio-religieux, il s’agit bien d'un cadre bâti pour une vie collective.

Tout autre est la conclusion qui se dégage de l'examen de la longue maison Dyak. Celle-ci, sur pilotis, peut avoir jusqu'à $400 \mathrm{~m}$ de longueur et comporte d'un côté du couloir central des pièces où habite une famille. De l'autre côté du couloir se situe une véranda mi-couverte sur laquelle est réservée une place pour chaque famille face à sa pièce. Tout se passe comme si une série de constructions avaient été accolées, créant un village sous un même toit 
Face à deux manières "d'habiter ", on peut émettre l'hypothèse qu'il existe deux séries dans l'aménagement de l'espace. Une série, que l'on nommerait tendance « cellulaire », comprendrait Pueblo Bonito ; les grandes maisons de l'Amérique du Sud, où chaque famille a un foyer et un espace de rangement sous un toit commun; et les villages de type Fang au Congo, où la construction est si exiguë qu'elle ne peut guère servir que pour s'abriter la nuit. L'autre comprendrait les constructions Dyak, les enclos africains à famille étendue, et toutes les maisons individuelles, série dont les représentants fournissent un cadre bâti complet pour la plus petite unité sociale.

Enfin, retenons deux méthodes, deux approches à l'étude de la construction. La première est celle de l'analyse de la circulation humaine inscrite sur le sol par le plan (Cresswell, 1966a).
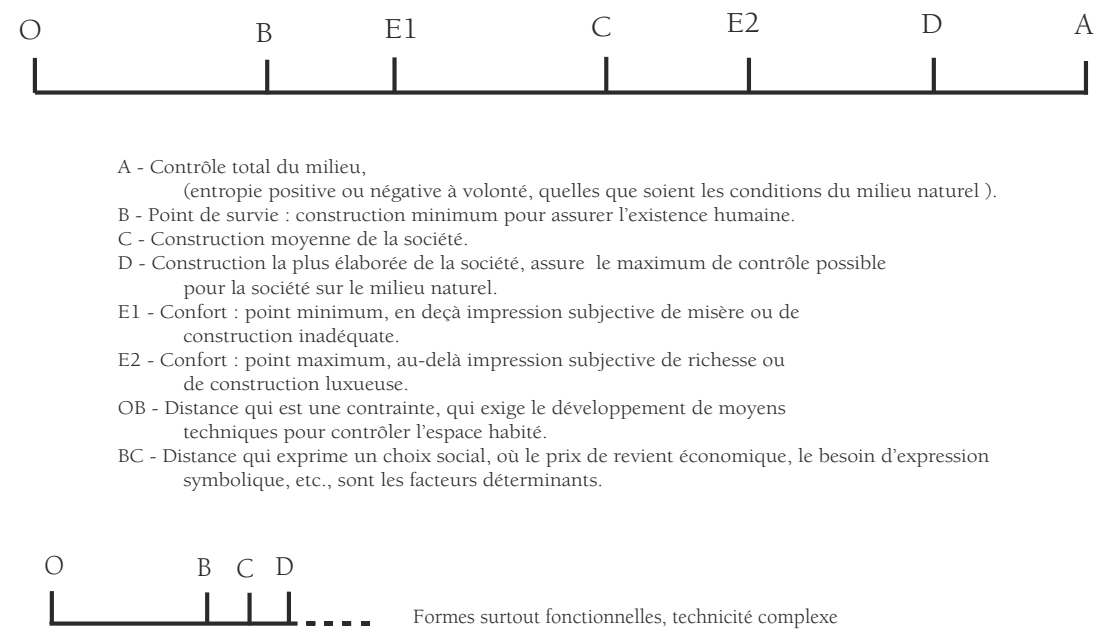

$$
\text { Esquimaux }
$$

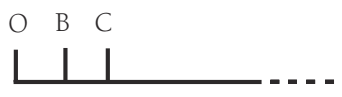

Formes surtout fonctionnelles, technicité simple

Australiens

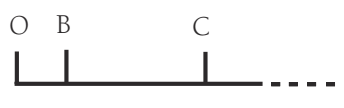

Formes sociologiques, symbolisme inportant, baute technicie Amazonie

\section{$[\ldots]$}

Un autre concept qui paraît fructueux est celui du degré d'indépendance d'une construction donnée par rapport au milieu naturel. Graphiquement, sur une échelle (fig. 4) est marqué un point zéro, qui représente une absence de construction, et un point A qui représente la création d'un micro-milieu totalement artificiel. Sur cette échelle est noté un point B qui signale le degré de construction nécessaire pour que l'homme survive dans un milieu naturel donné. Par exemple, dans les tropiques il sera minime, dans un milieu arctique il sera important. Le point $C$ représente la construction moyenne d'un groupe donné, et le point D la construction la plus élaborée, la plus indépendante

\section{Échelle de développement de l'habitation}

(Fig. 4) 
du milieu possible dans ce groupe. De part et d'autre du point $C$ se situent les points El et E2 représentant ce qu'on pourrait appeler une «zone de confort », c'est-à-dire qu'en dessous de El l'habitant aura l'impression de vivre dans l'inconfort, au-delà du point E2 il aura l'impression de vivre dans le luxe.

Si nous reprenons l'exemple tropical, le point $B$ et le point $O$ vont se situer très proches l'un de l'autre, tandis que si nous considérons les constructions, par exemple, des Indiens de l'Amazonie, extrêmement perfectionnées dans leur simplicité technique, nous éloignerons le point $C$ du point $\mathrm{O}$. Si nous faisons le même travail pour les Esquimaux, le point $B$ et le point $C$ seront tous deux éloignés du point $O$ et rapprochés l'un de l'autre. C'est dire que les constructions esquimaudes, igloo et tente, sont près du minimum nécessaire pour la survie, bien qu'étant aussi techniquement perfectionnées que les habitations amazoniennes. Nous pouvons déduire que dans le cas esquimau, la fonctionnalité prime dans les procédés techniques choisis par le constructeur, et nous en voyons un exemple dans la multifonctionnalité de la lampe esquimaude: séchoir, lumière, chauffage, foyer de cuisson des aliments. Par contre dans le cas amazonien les nécessités fonctionnelles de la construction sont minimes, un simple abri suffirait, et une recherche de perfectionnement technique est plutôt motivée par des raisons d'ordre sociologique.

[...Les procédés européens de construction poussent la zone de confort vers le côté droit sans rien changer aux conditions d'habiter traditionnelles...]

Le problème méthodologique posé par cette échelle est comment élaborer une combinatoire des qualités d'une maison afin de l'y situer. Les recherches se poursuivent sur ce point en tenant compte de facteurs tels que: température, humidité, insolation, insonorisation, mouvement de l'air; et probablement aussi de certains facteurs tels que la défense ou la durée de la construction avant que la réparation en devienne nécessaire.

\section{La dialectique technique et société}

Nous avons examiné explicitement ou implicitement jusqu'ici des cas d'influence des techniques ou des innovations techniques sur la société. Mais il ne faut pas oublier pour autant que la dialectique à laquelle il a été constamment fait référence dans ce qui précède, des rapports entre techniques et organisation sociale, doit rendre compte aussi de l'influence de la société sur l'innovation technique. Nous pouvons illustrer les deux aspects de ces relations avec un exemple irlandais. La substitution de la charrue à la bêche, qui représentait un bouleversement sur le plan purement technique, s'est accomplie sans mal dans la campagne irlandaise, tandis que le passage de la charrue tirée par un cheval à la charrue technique, s'est fait difficilement pour des raisons purement sociologiques.

[...A A milieu du XIXe siècle la population de l'Irlande comportait deux éléments: d'une part les Anglais, propriétaires fonciers, riches, possédant le pouvoir politique et protestant; et d'autre part les Irlandais, pauvres tenanciers fonciers, sans pouvoir politique et catholique. Dans l'ouest de l'Irlande surtout, le principal aliment était la pomme de terre semée dans des "lazy beds", des massifs de terre, et cultivées à la main. En 1845 la maladie détruisit la récolte des tubercules et provoqua les trois années de la Grande Famine. La forte émigration qui s'en suivit amena une baisse de la population de $50 \%$...] 
Dans ce milieu, que signifiait la substitution d'une charrue au travail à la bêche? En ce qui concerne l'effet mécanique sur le sol bêche et charrue sont similaires. Mais pour tout le reste la transformation était totale. Les planches que nous pourrions appeler horticoles sont transformées en planches à sillons. Le travail culturel dans son ensemble a dû changer car les labours à la charrue nécessitent un travail à la herse et au rouleau. Le comportement gestuel est différent. Et surtout tout un nouveau système d'échange de travail ou de coopération a dû s'établir. Mais le milieu social était favorable à ces transformations. Premièrement c'est à cette époque que se place la promulgation des «Land Acts » qui permettaient enfin aux paysans d'accéder à la propriété de la terre. Ensuite la très forte émigration des campagnes irlandaises a abouti à un manque de main-d'œuvre, d'où une recherche de meilleur rendement, recherche intensifiée par les lettres et les visites d'émigrés racontant l'Eldorado de l'Amérique. Par ailleurs, cette période est celle d'une intensification de la lutte nationaliste dont l'un des tenants est de sortir le paysan irlandais de la catégorie des «mere Irish » pour le faire rentrer dans, celle d'exploitant agricole. Enfin, une certaine amélioration du niveau de vie a permis l'achat de ce nouvel outil. Accession à la propriété de moyens de production, nécessité d'améliorer le rendement, valorisation de la condition irlandaise sont des motivations puissantes à l'innovation technique.

Qu'en est-il pour le passage de la charrue attelée à la charrue tractée? Il semblerait que les facteurs promouvant le changement soient encore plus favorables qu'au siècle passé. La main-d'œuvre manque toujours à la campagne irlandaise, l'émigration vers des pays lointains étant maintenant renforcée par l'émigration vers les villes. L'aide gouvernementale à la modernisation de l'agriculture et à la mise en valeur des fermes est considérable et va de l'analyse pédologique aux conseils de construction rurale en passant par l'achat de nouveaux matériels, surtout s'il s'agit de matériel mécanique. Les circonstances sociales, enfin, rehaussent le prestige du fermier moderne. Pourtant ce passage s'avère difficile. Il est vrai que le coût initial est important, mais celui-ci est récompensé par les prêts à long terme à des conditions très favorables. Il est vrai aussi que les champs dans l'ouest de l'Irlande sont souvent très petits, et de ce fait seul un remembrement dans certaines régions rendrait le tracteur réellement utilisable. Mais en fin de compte ce qui freine cette transformation est qu'une rentabilité satisfaisante du matériel agricole ne peut être réalisée par les fermiers irlandais qu'à l'intérieur de groupements coopératifs du genre CUMA. Or, ce type de groupement paraît aux yeux des fermiers comme le remplacement d'un réseau d'échanges séculaire par une propriété collective. On est prêt à rentrer dans une coopérative pour le traitement et la distribution de ses produits - les coopératives laitières en Irlande sont nombreuses. On est prêt à échanger son travail avec son voisin ou ses parents comme cela se faisait autrefois. On n'est pas prêt, et ceci d'autant moins que la lutte pour gagner la possession de ses propres terres est encore très récente dans les mémoires irlandaises, à renoncer aux fruits de cette lutte par une nouvelle forme de propriété. Ainsi, dans l'étude des techniques il ne faut jamais oublier le contexte social dans lequel celles-ci se pratiquent, et que les relations entre des phénomènes techniques et des manifestations socio-culturelles sont des relations à double sens.

La seule façon de conclure un essai qui cherche à poser les bases d'un programme de travail est d'indiquer quelques hypothèses de recherche, à la fois comme résumé des prémisses et comme illustration des directions de recherche à poursuivre. Le point qui me semble le plus important, et sur lequel il faudrait insister, est la nécessité d'élaborer des structures techniques. La société est structurée, nul ne le contestera, et 
si l'on veut étudier la place et l'influence des techniques dans cette société, il faut en analyser leur structure. L'outil conceptuel principal qui nous permettra de mettre en place ces structures est celui de chaîne opératoire ou, plutôt, de chaînes opératoires. Les problèmes qui se posent dans ce domaine sont ceux de la finesse de la description requise pour leur mise en place, de leur mode d'imbrication, et du codage de l'ensemble.

À ce concept de séries logiques de fabrication doit s'ajouter celui de la cohérence interne d'une chaîne opératoire. Par exemple, on ne trouvera pas un procédé entièrement automatisé comme l'une des étapes d'un processus dont toutes les autres étapes sont manuelles. De plus, le perfectionnement technique de cette chaîne - ou sa régression - dépendra certainement d'une plus efficace - ou moins bonne - utilisation de l'énergie. Enfin, au-delà d'une certaine amélioration, le progrès technique se réalise par paliers et non pas linéairement, en grande partie d'ailleurs, à cause de cette cohérence.

Les problèmes à résoudre ici sont aussi le codage et le mode d'imbrication des séries logiques, de même que la finesse d'analyse requise.

Une autre hypothèse est celle de la dialectique constante entre organisation sociale et techniques. Si les développements techniques peuvent fortement influencer les configurations sociales, celles-ci peuvent freiner voire empêcher le changement dans le domaine de la production. Les actions et réactions sont constantes et réciproques. Encore une fois le principal problème à résoudre semble bien être celui du codage dans ce cas des structures sociales, et il faut se souvenir, en procédant à ce travail, que les structures techniques et les structures sociales font partie d'une même structure générale, voire que l'une est contenue dans l'autre.

Pour terminer, soulignons que cette recherche revêt aussi un aspect pratique, car au fond la place et le contrôle des techniques dans la société humaine posent une question à laquelle il faudrait bien un jour se résoudre à répondre. Or, la connaissance précède toujours l'action. 


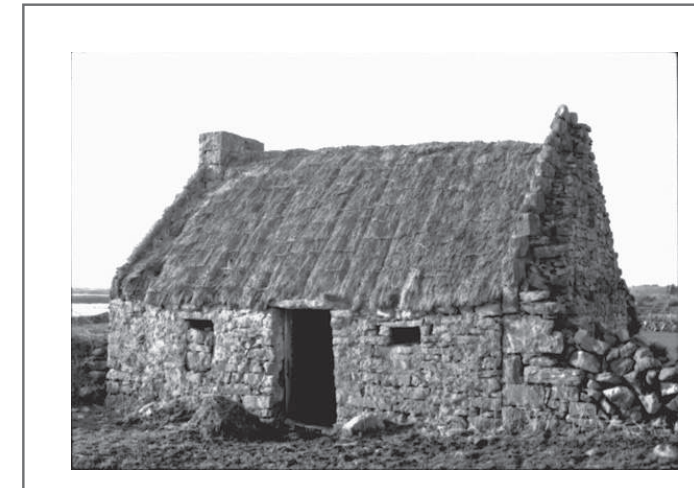

Cabane rurale en ruine du début du xixesiècle. Une chambre, une étable

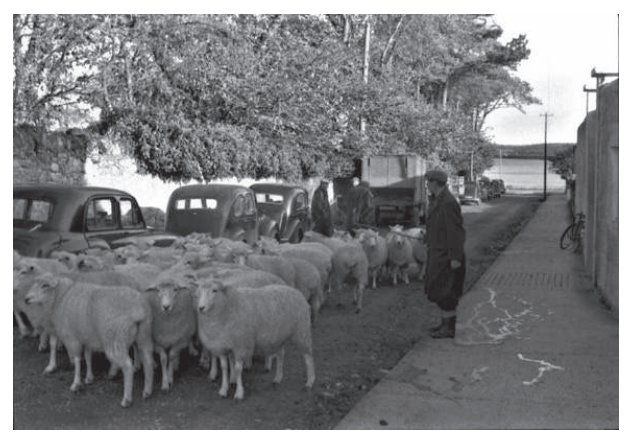

Ovins à la foire du 18 mai 1956.

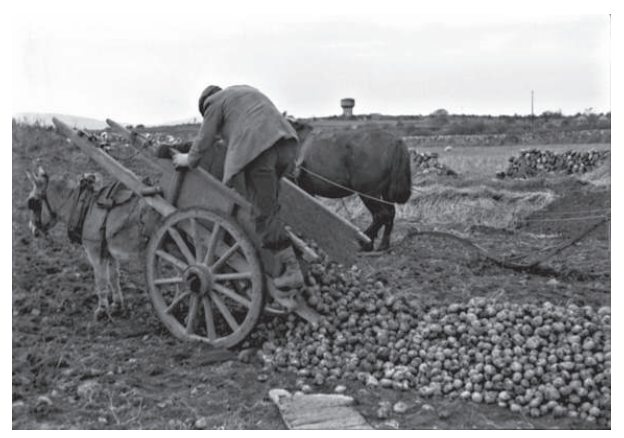

Une charette à deux roues facilite le travail dans des espaces exigus.

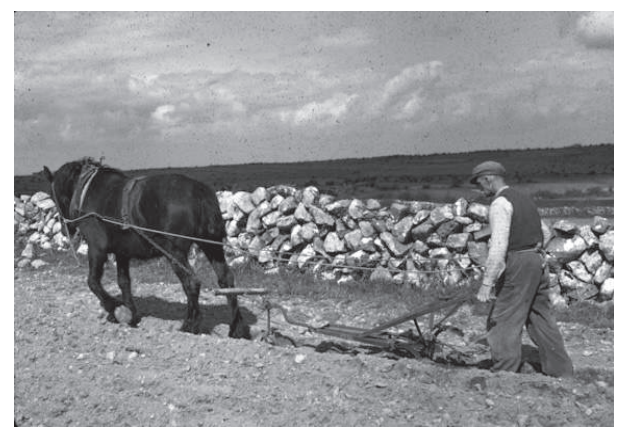

Ouverture des billons de pommes de terre avec une charrue sans avant train (swing plough).

Ces quatre photos et celles des forgerons irlandais de la page 26 sont reproduites avec l'aimable autorisation de The Robert Cresswell Archive, Don de Robert Cresswell. ${ }^{\oplus}$ Kinvara Community Council Ltd - Archives R. Cresswell 1956-1957. 


\section{NOTES}

1. Trente cinq ans après avoir écrit cette phrase, l'homme plus réaliste que je crois être devenu se sent obligé afin de se sentir moins honteux, de rappeler que cette « civilisation de loisir » est rendue possible en partie par le travail de pré-adolescents dans les mines du Chili... et ailleurs. Le poète John Donne a écrit de ne pas envoyer chercher pour qui sonne le glas, il sonne pour tous. Ajoutons que la honte est aussi pour nous.
2. Cf. White, 1962.

\section{RÉFERENCES}

Cresswell, R. 1965 Un Pressoir d'olives au Liban, essai de technologie comparée, L'Homme V-1.Paris.

- 1966 a Contribution ethnologique aux études architecturales de circulation, Études Rurales 21. Paris.

- 1966b Le Concept de structure au Proche Orient, Travaux et Jours 20. Beyrouth.

Godelier, M. 1966 Rationalité et irrationalité en économie. Paris : Maspéro.

Lee, R.- B. 1969 !kung Bushman subsistence an input-output analysis. In Andrew P. Vayda, Environment and cultural behavior, the natural history press. New York : Garden City.

Leroi-Gourhan, A. 1943 L'Homme et la matière. Paris : Albin Michel.

MacNeish, R. S. (ed.) 1967-1972 The Prehistory of the Tehuacan valley Vol. I-IV. Austin : University of Texas Press.

Perkins D. \& Daly P. 1968 A Hunters' village in neolithic Turkey, Scientific American 219 (5). New York.

Perrot J. 1969 Mallaha: les débuts de la vie sédentaire en Palestine il y a 10000 ans. In Atomes 261. Paris.

Pilcher, W. 1967 Some comments on the folk taxonomy of the Papago, American Anthropologist 69 (2), Wisconsin : Menasha.

Rappaport, Roy A 1969 Ritual regulation of environmental relations among a New Guinea People. In Andrew P. Vayda, Environment and cultural behavior, the natural history press. New York: Garden City.

White, L. 1962 Medieval technology and social change. Oxford : Clarendon Press.

Wittfogel, K. A. 1964 Le Despotisme oriental. Paris : Éditions de Minuit. 


\section{RÉSUMÉ}

En 1976 le CNRS créa un laboratoire pour étudier des phénomènes relevant du domaine de la technologie culturelle. L'équipe « Techniques et culture » qui vit le jour esquissa alors dans un premier bulletin un programme qui devait guider ses recherches dans les années à venir. La présente rédaction résume les grandes lignes de ce premier texte. Le regroupement des techniques en deux grandes divisions - celles qui « créent » des rapports sociaux, celles qui « reflètent » le fonctionnement et l'architecture des relations intra et intergroupes - est expliqué et justifié. Après un court rappel de l'épistémologie et de la méthodologie de l'enquête dans ce domaine, qui soulèvent entre autres les problèmes de l'innovation et de l'évolution, sont abordés quelques exemples de techniques « créatrices » tirées de l'époque médiévale: la charrue, l'étrier, le ferrage des animaux, l'assolement triennal, sous l'angle de leur influence sur les structures sociales. Puis l'habitation en tant qu'inscription de la société sur le sol permet de dégager des nouvelles avenues de recherche anthropologique. Enfin une dernière analyse compare le passage en Irlande de l'horticulture à la bêche à l'agriculture attelée, puis des animaux aux tracteurs d'où il ressort que parfois des facteurs sociaux revêtent autant d'importance que des critères purement techniques dans l'acceptation ou le refus de nouveautés.

\section{ABSTRACT}

In 1976 the CNRS (Centre national de la recherche scientifique) founded a laboratory for studying differents aspects of cultural technology. The work group « Techniques and culture » wich thus came into being laid down in its first research bulletin the general framework wich would be the cadre of its researches in the years to come. This article sums up the principal axes of that first text. The reasoning that explains and justifies, the grouping of techniques into two large divisions - those that « create » social relations, and those that mirror the architecture and the functioning of intra- and inter-relations within social groups - is analysed. After a short reminder of the epistemology and metholology obtaining in this domain wich among other things brings up the problems of innovation and evolution, a few examples from medieval Europe: the plough, the stirrup, iron shoes for animals, triennal crop rotatation, are examined from the point of view of their influence on social structure. The study of housing and urbanism (house grouping) as an imprinting of society on a geographical territory opens up news avenues of anthropological research. A final analysis compares the passage in, Ireland from horticulture with a spade to agriculture with harnessed animals, then from animals to tractors, from which it appears that social criteria are at least as important as purely technical ones as to the acceptance or denial of new vectors of change.

\section{MOTS CLÉS}

Séries historiques, séries logiques, invention, évolution, agriculture, artisanat.

\section{KEYWORDS}

Historical series, logical series, invention, evolution, agriculture, craft industry. 